\title{
Coefficient of orthogonal convexity of some Banach function spaces
}

\author{
by \\ Pawee Kolwicz (Poznań) and Stefan Rolewicz (Warszawa)
}

\begin{abstract}
We study orthogonal uniform convexity, a geometric property connected with property $(\beta)$ of Rolewicz, $P$-convexity of Kottman, and the fixed point property (see $[19,[20])$. We consider the coefficient of orthogonal convexity in Köthe spaces and Köthe-Bochner spaces.
\end{abstract}

1. Introduction. Let $\left(X,\|\cdot\|_{X}\right)$ be a real Banach space and $B(X), S(X)$ be the closed unit ball and unit sphere of $X$, respectively.

As usual, $\mathbb{N}, \mathbb{R}$ and $\mathbb{R}_{+}$stand for the sets of natural, real and nonnegative real numbers, respectively. Let $(T, \Sigma, \mu)$ be a measure space with a $\sigma$-finite, complete measure $\mu$, and $\left(\mathbb{N}, 2^{\mathbb{N}}, m\right)$ be the counting measure space. By $L^{0}=L^{0}(T)$ we denote the set of all $\mu$-equivalence classes of real-valued measurable functions defined on $T$, and by $l^{0}=l^{0}(m)$ the linear space of all real sequences.

Definition 1. A Banach space $E=\left(E,\|\cdot\|_{E}\right)$ is said to be a Köthe space if $E$ is a linear subspace of $L^{0}$ and:

(i) if $x \in E, y \in L^{0}$, and $|y| \leq|x| \mu$-a.e., then $y \in E$ and $\|y\|_{E} \leq\|x\|_{E}$,

(ii) there exists a function $x$ in $E$ that is positive on the whole $T$ (see [17] and [23]).

Every Köthe space is a Banach lattice in the obvious order $(x \geq 0$ if $x(t) \geq 0$ for $\mu$-a.e. $t \in T)$. In particular, if $\mu$ is non-atomic, then we shall say that $E$ is a Köthe function space, while $(T, \Sigma, \mu)=\left(\mathbb{N}, 2^{\mathbb{N}}, m\right)$, then $E$ is a Köthe sequence space. In the last case we denote by $e_{i}=(0, \ldots, 0,1,0, \ldots)$ the $i$ th unit vector.

A Köthe space $E$ is said to be:

2000 Mathematics Subject Classification: 46E30, 46E40, 46B20.

Key words and phrases: Köthe-Bochner space, Orlicz space, uniform convexity, uniform monotonicity, orthogonal uniform convexity.

Research of P. Kolwicz supported by Foundation of Polish Science, scholarship 2002. 
- strictly monotone $(E \in(\mathrm{SM}))$ if for every $0 \leq y \leq x$ with $y \neq x$ we have $\|y\|_{E}<\|x\|_{E}$

- uniformly monotone $(E \in(\mathrm{UM}))$ if for every $q \in(0,1)$ there exists $p \in(0,1)$ such that for all $0 \leq y \leq x$ satisfying $\|x\|_{E} \leq 1$ and $\|y\|_{E} \geq q$ we have $\|x-y\|_{E} \leq 1-p$ (see [4]);

- order continuous $(E \in(\mathrm{OC}))$ if for every $x \in E$ and every sequence $\left(x_{m}\right)$ in $E$ such that $0 \leq x_{m} \leq|x|$ and $x_{m} \rightarrow 0 \mu$-a.e. we have $\left\|x_{m}\right\|_{E} \rightarrow$ 0 (see [17] and [23]).

It is known that if $E \in(\mathrm{UM})$, then $E \in(\mathrm{OC})$ (see [8, Proposition 2.1]).

We study a geometric property called orthogonal uniform convexity $\left(\mathrm{UC}^{\perp}\right)$. It was introduced in [19] in the study of property $(\beta)$ of Rolewicz. Although the original definition of property $\mathrm{UC}^{\perp}$ is based on the unit ball $B(E)$ of $E$ (see [19]), we can equivalently use the unit sphere $S(E)$.

The notation $r \vee s=\max \{r, s\}, r \wedge s=\min \{r, s\}$ for any $r, s \in \mathbb{R}$ and $A \div B=(A \backslash B) \cup(B \backslash A)$ for $A, B \in \Sigma$ will be used.

Definition 2. A Köthe space $\left(E,\|\cdot\|_{E}\right)$ is orthogonally uniformly convex $\left(E \in\left(\mathrm{UC}^{\perp}\right)\right)$ if for each $\varepsilon>0$ there is $\delta=\delta(\varepsilon)>0$ such that for any $x, y \in S(E)$,

$$
\left\|x \chi_{A_{x y}}\right\|_{E} \vee\left\|y \chi_{A_{x y}}\right\|_{E} \geq \varepsilon \text { implies }\|(x+y) / 2\|_{E} \leq 1-\delta,
$$

where $A_{x y}=\operatorname{supp} x \div \operatorname{supp} y$.

We denote by $\delta_{E}^{\perp}(\varepsilon)$ the modulus of orthogonal convexity and by $\varepsilon_{0}^{\perp}(E)$ the coefficient of orthogonal convexity of the space $E$, defined by

$$
\begin{aligned}
\delta_{E}^{\perp}(\varepsilon) & =\inf \left\{1-\|(x+y) / 2\|_{E}: x, y \in S(E),\left\|x \chi_{A_{x y}}\right\|_{E} \vee\left\|y \chi_{A_{x y}}\right\|_{E} \geq \varepsilon\right\}, \\
\varepsilon_{0}^{\perp}(E) & =\sup \left\{\varepsilon \geq 0: \delta_{E}^{\perp}(\varepsilon)=0\right\} .
\end{aligned}
$$

Clearly, $\delta_{E}^{\perp}$ maps $[0,1]$ into $[0,1]$ is nondecreasing; moreover, $E \in\left(\mathrm{UC}^{\perp}\right)$ if and only if $\varepsilon_{0}^{\perp}(E)=0$. It is also easy to see that $\varepsilon_{0}^{\perp}(E)=1$ for $E \in$ $\left\{L^{1}, L^{\infty}, l^{1}, c_{0}\right\}$.

Recall that a Banach space $X$ is said to be uniformly convex $(X \in(\mathrm{UC})$ ) if for each $\varepsilon>0$ there is $\delta>0$ such that for any $x, y \in S(X)$ the inequality $\|x-y\|_{X}>\varepsilon$ implies $\|x+y\|_{X}<2(1-\delta)$ (see [7]).

Obviously, if $E \in(\mathrm{UC})$, then $E \in\left(\mathrm{UC}^{\perp}\right)$. It is known that every uniformly convex Köthe space is uniformly monotone (see [11]). Moreover,

Lemma 1 ([19, Lemma 3]). If $E \in\left(\mathrm{UC}^{\perp}\right)$, then $E \in(\mathrm{UM})$.

The converse of Lemma 1 is not true as the examples of $L^{1}, l^{1}$ show.

There are numerous geometric properties lying between uniform convexity and reflexivity. The $P$-convexity of Kottman is one of such properties (see [22]). Recall that $X$ is said to be $P$-convex if $P(n, X)<1 / 2$ for 
some positive integer $n$, where $P(n, X)=\sup \{r>0$ : there exist $n$ disjoint balls of radius $r$ in $B(X)\}$ (see [22]). Although orthogonal uniform convexity is much weaker than uniform convexity (it need not even imply strict convexity), it is still stronger than $P$-convexity (see [20]). Let us also recall that $X$ is called $B$-convex provided it is uniformly non- $l_{n}^{1}$ for some $n \in \mathbb{N}$, i.e. there exists $\delta>0$ such that for all $x_{1}, \ldots, x_{n} \in B(X)$ we have $\left\|x_{1} \pm x_{2} \pm \cdots \pm x_{n}\right\|_{X} \leq n(1-\delta)$ for some choice of signs (see [22]). Geometrically, a uniformly non- $l_{n}^{1}$ space is one which does not have $n$-dimensional subspaces whose norms are arbitrarily good approximations of the $l^{1}$ norm. It is known that every $B$-convex and uniformly monotone Köthe space has the fixed point property for nonexpansive self-maps on closed bounded convex sets (see [1]). Note also that a $P$-convex Banach space is $B$-convex (see $[22]$ ). Consequently, by the above arguments and Lemma 1 , the fixed point property follows from orthogonal uniform convexity.

Another important geometric property lying between uniform convexity and reflexivity is property $(\beta)$ of Rolewicz. Although it was introduced in the study of well-posed problems in optimization theory (see [25], [26]), it has been widely investigated from the geometric point of view (see [19] and [20] for references). It is known that in Köthe sequence spaces one has the implications (UC) $\Rightarrow\left(\mathrm{UC}^{\perp}\right) \Rightarrow(\beta)$ and none of them can be reversed in general (see [20]). However, property $(\beta)$ and $\left(\mathrm{UC}^{\perp}\right)$ coincide in Orlicz sequence spaces (see $[20]$ ) and more generally in symmetric Köthe sequence spaces (see [21]). On the other hand, the implications $(\mathrm{UC}) \Rightarrow(\beta) \Rightarrow\left(\mathrm{UC}^{\perp}\right)$ hold in Köthe function spaces and the last one cannot be reversed (see [19], $[20])$.

In this paper we consider the coefficient $\varepsilon_{0}^{\perp}$ of orthogonal convexity in Köthe spaces, Orlicz spaces and Köthe-Bochner spaces. Analogous investigations for the classical coefficient $\varepsilon_{0}$ of convexity have been carried out in [12] and [13]. We have taken some inspirations from those papers.

\section{Results}

2.1. Köthe spaces. In this section we prove that a Köthe space with $\varepsilon_{0}^{\perp}(E)<1$ must be superreflexive. First we need to recall the notion of upper and lower $p$-estimates.

Let $1<p<\infty$. A Köthe space $E$ is said to satisfy an upper, respectively lower, p-estimate (for disjoint elements) if there exists a constant $M<\infty$ such that, for every choice of pairwise disjoint elements $\left\{x_{i}\right\}_{i=1}^{n}$ in $E$, we have

$$
\left\|\sum_{i=1}^{n} x_{i}\right\|_{E} \leq M\left(\sum_{i=1}^{n}\left\|x_{i}\right\|_{E}^{p}\right)^{1 / p}, \text { resp. },\left\|\sum_{i=1}^{n} x_{i}\right\|_{E} \geq M^{-1}\left(\sum_{i=1}^{n}\left\|x_{i}\right\|_{E}^{p}\right)^{1 / p}
$$

(see $[23])$. 
THEOREM 1. Let $E$ be a Köthe space. If $\varepsilon_{0}^{\perp}(E)<1$, then $E$ is superreflexive.

Proof. Suppose that $E$ is not superreflexive. Then either $E$ satisfies an upper $p$-estimate for no $p>1$, or $E$ satisfies a lower $q$-estimate for no $q<\infty$. Indeed, otherwise, by [23, Theorem 1.f.7], $E$ satisfies an upper $p_{0}$-estimate and a lower $q_{0}$-estimate for some $1<p_{0}<2<q_{0}$ (see also the diagram in [23, p. 101]) and consequently [23, Theorem 1.f.10] shows that $E$ can be given an equivalent uniformly convex norm, contrary to James's characterization of superreflexivity [9, Theorem 5.1].

Now, by [23, Theorem 1.f.12], either for every $\varepsilon>0$ there are disjoint elements $x_{1}, x_{2}$ in $E$ such that

$$
(1-\varepsilon)\left(\left|a_{1}\right|+\left|a_{2}\right|\right) \leq\left\|a_{1} x_{1}+a_{2} x_{2}\right\|_{E} \leq\left|a_{1}\right|+\left|a_{2}\right|
$$

for all scalars $a_{1}, a_{2}$, or for every $\varepsilon>0$ there are disjoint $y_{1}, y_{2}$ in $E$ such that

$$
\left|a_{1}\right| \vee\left|a_{2}\right| \leq\left\|a_{1} y_{1}+a_{2} y_{2}\right\|_{E} \leq(1+\varepsilon)\left(\left|a_{1}\right| \vee\left|a_{2}\right|\right)
$$

for all scalars $a_{1}, a_{2}$. We assume that (2) holds, because in the case of (1) the proof is analogous and simpler (it is enough to take $x=x_{1}$ and $y=x_{2}$ from the proof below). Set

$$
x=\frac{y_{1}+y_{2}}{\left\|y_{1}+y_{2}\right\|_{E}}, \quad y=\frac{y_{2}}{\left\|y_{2}\right\|_{E}} .
$$

Putting $a_{1}=\left\|y_{2}\right\|_{E}, a_{2}=\left\|y_{2}\right\|_{E}+\left\|y_{1}+y_{2}\right\|_{E}$ and applying (2) we have

$$
\begin{aligned}
\left\|\frac{x+y}{2}\right\|_{E} & =\frac{1}{2}\left\|\frac{y_{1}+y_{2}}{\left\|y_{1}+y_{2}\right\|_{E}}+\frac{y_{2}}{\left\|y_{2}\right\|_{E}}\right\|_{E}=\frac{1}{2\left\|y_{1}+y_{2}\right\| E\left\|y_{2}\right\|_{E}}\left[a_{1} y_{1}+a_{2} y_{2}\right]_{E} \\
& \geq \frac{1}{2\left\|y_{1}+y_{2}\right\|_{E}\left\|y_{2}\right\|_{E}} \max \left\{a_{1}, a_{2}\right\}=\frac{1}{2}\left(\frac{1}{\left\|y_{1}+y_{2}\right\|_{E}}+\frac{1}{\left\|y_{2}\right\|_{E}}\right) \\
& \geq \frac{1}{\left\|y_{1}+y_{2}\right\|_{E}} \geq \frac{1}{1+\varepsilon} .
\end{aligned}
$$

Moreover

$$
\left\|x \chi_{A}\right\|_{E}=\frac{\left\|y_{1}\right\|_{E}}{\left\|y_{1}+y_{2}\right\|_{E}} \geq \frac{1}{1+\varepsilon}, \quad \text { where } \quad A=\operatorname{supp} x \div \operatorname{supp} y .
$$

Hence $\delta_{E}^{\perp}(1 /(1+\varepsilon)) \leq 1-1 /(1+\varepsilon) \leq \varepsilon$. Now, given $\gamma<1$, we have $\delta_{E}^{\perp}(\gamma) \leq \delta_{E}^{\perp}(1 /(1+\varepsilon)) \leq \varepsilon$ for each $\varepsilon \in(0,1 / \gamma-1)$. Hence $\delta_{E}^{\perp}(\gamma)=0$. This means that $\varepsilon_{0}^{\perp}(E)=1$.

REmark 1. The converse of Theorem 1 is not true. The simplest example of a superreflexive Köthe space $E$ with $\varepsilon_{0}^{\perp}(E)=1$ is $l_{2}^{\infty}$ or $l_{2}^{1}$ (a two-dimensional $l^{\infty}$ or $l^{1}$ ). We will also give an analogous example of an infinite-dimensional Köthe space (see Corollary 1 below). 
REMARK 2. Note that $\varepsilon_{0}^{\perp}(E) \in[0,1]$ and for the classical coefficient of convexity $\varepsilon_{0}(X)$ of a Banach space $X$ we have $\varepsilon_{0}(X) \in[0,2]$ (see [9], [13] and [23]). Recall that $X$ is called uniformly non-square if $\varepsilon_{0}(X)<2$ (see [15]). Combining the results of James and Enflo we conclude that a Banach space $X$ is superreflexive iff $X$ has an equivalent uniformly non-square norm (see [9, Theorem 5.1]). Then Theorem 1 is, in a sense, analogous to the James and Enflo theorem.

2.2. Orlicz spaces. In this section we estimate the coefficient $\varepsilon_{0}^{\perp}$ of orthogonal convexity of Orlicz spaces. As a corollary we conclude that the converse of Theorem 1 is not true in general. First we need to recall some terminology.

We say that $\Phi: \mathbb{R} \rightarrow[0, \infty]$ is an Orlicz function if $\Phi(0)=0, \Phi$ is convex, even, left continuous on $[0, \infty)$, and not identically zero or infinity.

For every Orlicz function $\Phi$ we define the complementary function $\Phi^{*}$ by the formula $\Phi^{*}(v)=\sup _{u>0}\{u|v|-\Phi(u)\}$ for every $v \in \mathbb{R}$.

By the Orlicz function space $L_{\Phi}(\mu)$ we mean

$$
L_{\Phi}(\mu)=\left\{x \in L^{0}: I_{\Phi}(c x)=\int_{T} \Phi(c x(t)) d \mu<\infty \text { for some } c>0\right\} .
$$

Similarly we define the Orlicz sequence space $l_{\Phi}$ by

$$
l_{\Phi}=\left\{x \in l^{0}: I_{\Phi}(c x)=\sum_{i=1}^{\infty} \Phi(c x(i))<\infty \text { for some } c>0\right\} .
$$

We equip $L_{\Phi}(\mu)$ and $l_{\Phi}$ with the Nakano-Luxemburg norm defined by

$$
\|x\|_{\Phi}=\inf \left\{\varepsilon>0: I_{\Phi}(x / \varepsilon) \leq 1\right\} .
$$

We say that an Orlicz function $\Phi$ satisfies the $\Delta_{2}$-condition for all $u$ (for large $u)$ [for small $u$ ] if there is a constant $k>2$ (there are $u_{0}>0$ with $\Phi\left(u_{0}\right)<\infty$ and $k>2$ ) [there are $u_{0}>0$ with $\Phi\left(u_{0}\right)>0$ and $k>2$ ] such that

$$
\Phi(2 u) \leq k \Phi(u)
$$

for every $u \in \mathbb{R}$ (for every $|u| \geq u_{0}$ ) [for every $|u| \leq u_{0}$ ], respectively. We shall write $\Phi \in \Delta_{2}^{a}, \Phi \in \Delta_{2}^{l}, \Phi \in \delta_{2}$ if $\Phi$ satisfies the $\Delta_{2}$-condition for all $u$, for large $u$, for small $u$, respectively.

For more details we refer to [6] and [24].

Remark 3. Note that if $\Phi \in \delta_{2}$, then $\Phi\left(u_{0}\right)<\infty$. Moreover, in the definition of the $\Delta_{2}$-condition for small $u$ we cannot omit the assumption that $\Phi\left(u_{0}\right)>0$, because without it the $\Delta_{2}$-condition would not guarantee that $l_{\Phi}$ is order continuous, as it should be. Indeed, if $\Phi\left(u_{0}\right)=0$, then $l_{\Phi}=l^{\infty}$ as sets and they are isomorphic. Consequently, since $l^{\infty}$ is not (OC), neither is $l_{\Phi}$. On the other hand, we have $\Phi(2 u)=k \Phi(u)=0$ for every $u \in\left[0, u_{0} / 2\right]$. 
Similarly, if $\Phi \in \Delta_{2}^{l}$, then $\Phi\left(u_{0}\right)>0$, and in the definition of the $\Delta_{2^{-}}$ condition for large $u$ the assumption that $\Phi\left(u_{0}\right)<\infty$ cannot be omitted.

We shall use the following constants:

$$
\begin{gathered}
a_{\Phi}=\sup \{u \geq 0: \Phi(u)=0\}, \quad b_{\Phi}=\sup \{u \geq 0: \Phi(u)<\infty\}, \\
\alpha_{\Phi}=\sup \{u \geq 0: \Phi \text { is linear in }[0, u]\} .
\end{gathered}
$$

Notice that if $\Phi \in \Delta_{2}^{a}$, then $a_{\Phi}=0$ and $b_{\Phi}=\infty$. If $\Phi \in \Delta_{2}^{l}$, then $b_{\Phi}=\infty$. If $\Phi \in \delta_{2}$, then $a_{\Phi}=0$.

To prove our main results we shall need some auxiliary lemmas. The next lemma can be easily deduced from [2, Lemma 2].

Lemma 2. If $\Phi^{*} \in \Delta_{2}^{l}$, then for every $w>\alpha_{\Phi}$ there exists $\gamma=\gamma(w) \in$ $(0,1)$ such that $\Phi(u / 2) \leq(1-\gamma) \Phi(u) / 2$ for all $u \geq w$ satisfying $\Phi(u)<\infty$.

Lemma $3([18$, Lemma 3$])$. If $\Phi^{*} \in \delta_{2}$, then for every $w>0$ with $0<$ $\Phi(w)<\infty$ there exists $\gamma=\gamma(w) \in(0,1)$ such that $\Phi(u / 2) \leq(1-\gamma) \Phi(u) / 2$ for all $u \leq w$.

The next lemma was proved in [10] in the general case.

Lemma 4. Let $\Phi \in \Delta_{2}^{l}$ and $L_{\Phi}(\mu)$ be the Orlicz function space over a finite measure space. Then:

(a) For every sequence $\left(x_{n}\right)$ in $L_{\Phi}(\mu)$ the equivalence $\left\|x_{n}\right\|_{\Phi} \rightarrow 0 \Leftrightarrow$ $I_{\Phi}\left(x_{n}\right) \rightarrow 0$ holds if and only if $\Phi$ vanishes only at zero.

(b) For every $p \in(0,1)$ there exists $q \in(0,1)$ such that for any $x \in$ $L_{\Phi}(\mu)$ the inequality $I_{\Phi}(x) \leq 1-p$ implies $\|x\|_{\Phi} \leq 1-q$.

Lemma 5. Let $\Phi \in \delta_{2}$ and let $b_{\Phi}$ be as defined in (3). Then:

(a) For every sequence $\left(x_{n}\right)$ in $l_{\Phi}$ we have $\left\|x_{n}\right\|_{\Phi} \rightarrow 0$ if and only if $I_{\Phi}\left(x_{n}\right) \rightarrow 0$.

(b) Suppose that $\Phi\left(b_{\Phi}\right)<1$. Then for every $\sigma, p \in(0,1)$ there exists $q=q(\sigma, p) \in(0,1)$ such that for any $x \in A$ with $I_{\Phi}(x) \leq 1-p$ we have $\|x\|_{\Phi} \leq 1-q$, where $A=\left\{x \in l_{\Phi}:|x(i)|<(1-\sigma) b_{\Phi}\right.$ for each $\left.i\right\}$.

Proof. (a) It is known that $\left\|x_{n}\right\|_{\Phi} \rightarrow 0$ if and only if $I_{\Phi}\left(\eta x_{n}\right) \rightarrow 0$ for any $\eta>0$. Since $\Phi \in \delta_{2}$, this completes the proof.

(b) This was proved in [16] in the general case, but with the assumption that $b_{\Phi}=\infty$. We point out only the necessary changes to that proof. Let $p, \sigma \in(0,1)$. Take $\delta=\sigma /(1-\sigma)>0$. Then the inequality $u \leq(1-\sigma) b_{\Phi}$ implies $(1+\delta) u \leq b_{\Phi}$. Consequently, since $\Phi \in \delta_{2}$, there exists $k_{0}>0$ such that $\Phi((1+\delta) u) \leq k_{0} \Phi(u)$ for every $|u| \leq b_{\Phi} /(1+\delta)$. Then the proof can be finished as in [16, Lemma 9].

Note that the case $\Phi\left(b_{\Phi}\right) \geq 1$ was handled in [20, Lemma 4c]. 
Given an Orlicz function $\Phi$ with $a_{\Phi}=0$ we define

$$
f_{\Phi}(u)=\sup _{v>0} \frac{\Phi(u v)}{\Phi(v)} .
$$

Applying [14, Lemma 1(i)] we immediately obtain

Lemma 6. Assume that $\Phi \in \Delta_{2}^{a}$. Then for any $a \in(0,1)$ and $x \in L_{\Phi}(\mu)$ we have the implication

$$
\|x\|_{\Phi} \geq a \Rightarrow I_{\Phi}(x) \geq 1 / f_{\Phi}(1 / a) .
$$

Theorem 2. (I) Suppose that $\mu$ is non-atomic and infinite. Then:

1. $\varepsilon_{0}^{\perp}\left(L_{\Phi}(\mu)\right)=1$ whenever $\Phi \notin \Delta_{2}^{a}$ or $\Phi^{*} \notin \Delta_{2}^{a}$.

2. If $\Phi \in \Delta_{2}^{a}$ and $\Phi^{*} \in \Delta_{2}^{a}$, then $\varepsilon_{0}^{\perp}\left(L_{\Phi}(\mu)\right)=0$.

(II) Assume that $\mu$ is non-atomic and finite. Let $a_{\Phi}$ and $\alpha_{\Phi}$ be as defined in (3) and (4), respectively.

1. $\varepsilon_{0}^{\perp}\left(L_{\Phi}(\mu)\right)=1$ if $\Phi \notin \Delta_{2}^{l}$ or $\Phi^{*} \notin \Delta_{2}^{l}$.

2. Suppose $\Phi \in \Delta_{2}^{l}, \Phi^{*} \in \Delta_{2}^{l}$ and $a_{\Phi}=0$. Then:

(a) If $\alpha_{\Phi}=0$, then $\varepsilon_{0}^{\perp}\left(L_{\Phi}(\mu)\right)=0$.

(b) If $\alpha_{\Phi}>0$, then $\varepsilon_{0}^{\perp}\left(L_{\Phi}(\mu)\right) \geq \min \left\{1, \Phi\left(\alpha_{\Phi}\right) \mu(T) / 2\right\}$. In particular, if $\Phi\left(\alpha_{\Phi}\right) \mu(T) \geq 2$, then $\varepsilon_{0}^{\perp}\left(L_{\Phi}(\mu)\right)=1$.

(c) If $\alpha_{\Phi}>0$ and $\Phi\left(\alpha_{\Phi}\right) \mu(T)<1$, then

$$
\varepsilon_{0}^{\perp}\left(L_{\Phi}(\mu)\right) \in\left[\Phi\left(\alpha_{\Phi}\right) \mu(T) / 2, u_{1}\right]
$$

where $u_{1}=1 / f_{\Phi}^{-1}\left(1 / \Phi\left(\alpha_{\Phi}\right) \mu(T)\right)$.

3. Assume that $\Phi \in \Delta_{2}^{l}$, $\Phi^{*} \in \Delta_{2}^{l}$ and $a_{\Phi}>0$. Then $\varepsilon_{0}^{\perp}\left(L_{\Phi}(\mu)\right) \geq u_{2}$, where $u_{2}=\left\|a_{\Phi} \chi_{T}\right\|_{\Phi}$.

Proof. (I.1) If $\Phi \notin \Delta_{2}^{a}$ or $\Phi^{*} \notin \Delta_{2}^{a}$, then $L_{\Phi}(\mu)$ is not reflexive, hence, by Theorem 1, we get $\varepsilon_{0}^{\perp}\left(L_{\Phi}(\mu)\right)=1$.

(I.2) Since $\Phi \in \Delta_{2}^{a}$ and $\Phi^{*} \in \Delta_{2}^{a}$, [20, Theorem 4a] yields $L_{\Phi}(\mu) \in\left(\mathrm{UC}^{\perp}\right)$, hence $\varepsilon_{0}^{\perp}\left(L_{\Phi}(\mu)\right)=0$.

(II.1) If $\Phi \notin \Delta_{2}^{l}$ or $\Phi^{*} \notin \Delta_{2}^{l}$, then $L_{\Phi}(\mu)$ is not reflexive, and so $\varepsilon_{0}^{\perp}\left(L_{\Phi}(\mu)\right)=1$ by Theorem 1 .

(II.2a) If $\alpha_{\Phi}=0$, then, by [20, Theorem 4b], $L_{\Phi}(\mu) \in\left(\mathrm{UC}^{\perp}\right)$, hence $\varepsilon_{0}^{\perp}\left(L_{\Phi}(\mu)\right)=0$.

(II.2b) We consider two cases.

A. Suppose that $\Phi\left(\alpha_{\Phi}\right) \mu(T) \geq 2$. Then there exist measurable disjoint sets $T_{1}, T_{2}$ with $\mu\left(T_{1}\right)=\mu\left(T_{2}\right)$ and a number $u_{0} \leq \alpha_{\Phi}$ such that $\Phi\left(u_{0}\right) \mu\left(T_{1}\right)=1$. Define

$$
x=u_{0} \chi_{T_{1}}, \quad y=u_{0} \chi_{T_{2}} .
$$

Then $I_{\Phi}(x)=I_{\Phi}(y)=1$. Hence $\|x\|_{\Phi}=\|y\|_{\Phi}=1$. Similarly, $\left\|x \chi_{A}\right\|_{\Phi}=1$, where $A=\operatorname{supp} x \div \operatorname{supp} y$, and $\|(x+y) / 2\|_{\Phi}=1$. Thus $\varepsilon_{0}^{\perp}\left(L_{\Phi}(\mu)\right)=1$. 
B. Assume that $\Phi\left(\alpha_{\Phi}\right) \mu(T)<2$. Let $\varepsilon>0$. Take disjoint $T_{1}, T_{2} \in \Sigma$ such that $\mu\left(T_{1}\right)=\mu\left(T_{2}\right)$ and $(\mu(T)-\varepsilon) / 2<\mu\left(T_{1}\right)<\mu(T) / 2$. Let $T_{3} \subset T \backslash\left(T_{1} \cup T_{2}\right)$ with $\mu\left(T_{3}\right)>0$. Since $\Phi \in \Delta_{2}^{l}$, we have $b_{\Phi}=\infty$. Thus there is $\beta>0$ with $\Phi\left(\alpha_{\Phi}\right) \mu\left(T_{1}\right)+\Phi(\beta) \mu\left(T_{3}\right)=1$. Define

$$
x=\alpha_{\Phi} \chi_{T_{1}}+\beta \chi_{T_{3}}, \quad y=\alpha_{\Phi} \chi_{T_{2}}+\beta \chi_{T_{3}} .
$$

Then $\|x\|_{\Phi}=\|y\|_{\Phi}=1=\|(x+y) / 2\|_{\Phi}$. Moreover, since $\Phi\left(\alpha_{\Phi}\right) \mu\left(T_{1}\right)<1$, setting $A=\operatorname{supp} x \div \operatorname{supp} y$, we get

$$
I_{\Phi}\left(\frac{x \chi_{A}}{\Phi\left(\alpha_{\Phi}\right) \mu\left(T_{1}\right)}\right) \geq 1
$$

Hence

$$
\left\|x \chi_{A}\right\|_{\Phi} \geq \Phi\left(\alpha_{\Phi}\right) \mu\left(T_{1}\right)>\Phi\left(\alpha_{\Phi}\right)(\mu(T)-\varepsilon) / 2 .
$$

Then $\delta_{L_{\Phi}(\mu)}^{\perp}\left(\Phi\left(\alpha_{\Phi}\right)(\mu(T)-\varepsilon) / 2\right)=0$, so $\varepsilon_{0}^{\perp}\left(L_{\Phi}(\mu)\right) \geq \Phi\left(\alpha_{\Phi}\right) \mu(T) / 2$, because $\varepsilon>0$ is arbitrary.

(II.2c) Since $\Phi \in \Delta_{2}^{l}$ and $a_{\Phi}=0$ and $\alpha_{\Phi}>0$, we have $\Phi \in \Delta_{2}^{a}$. Thus $f_{\Phi}$ is finite-valued. Applying case (II.2.b) we get $\varepsilon_{0}^{\perp}\left(L_{\Phi}(\mu)\right) \geq \Phi\left(\alpha_{\Phi}\right) \mu(T) / 2$. First we note that $\Phi\left(\alpha_{\Phi}\right) \mu(T) / 2<u_{1}<1$. Indeed, since $f_{\Phi}$ is convex, $f_{\Phi}^{-1}$ is concave, and consequently $f_{\Phi}^{-1}(u) \leq u$ for any $u \geq 1$. Then $u_{1}>$ $\Phi\left(\alpha_{\Phi}\right) \mu(T) / 2$. Moreover, $f_{\Phi}^{-1}(1)=1$ and $f_{\Phi}$ is strictly increasing on $\mathbb{R}_{+}$. Hence $u_{1}<1$.

Let $a>u_{1}$. Then there are $\eta>0$ and $\alpha_{1}>\alpha_{\Phi}$ such that $u_{1}<u_{0}<a$, where $u_{0}=1 / f_{\Phi}^{-1}\left(1 /(1+\eta) \Phi\left(\alpha_{1}\right) \mu(T)\right)$, because $\Phi$ and $f_{\Phi}^{-1}$ are continuous and strictly increasing. Let $x, y \in S\left(L_{\Phi}(\mu)\right)$ be such that $\left\|x \chi_{A}\right\|_{\Phi} \vee\left\|y \chi_{A}\right\|_{\Phi} \geq$ $a$, where $A=\operatorname{supp} x \div \operatorname{supp} y$. Without loss of generality we may assume that $\left\|x \chi_{A}\right\|_{\Phi} \geq a$. Lemma 6 implies $I_{\Phi}\left(x \chi_{A}\right) \geq(1+\eta) \Phi\left(\alpha_{1}\right) \mu(T)>0$. Define

$$
A_{1}=\left\{t \in A:|x(t)| \geq \alpha_{1}\right\} .
$$

Then

$$
\begin{aligned}
I_{\Phi}\left(x \chi_{A_{1}}\right) & =I_{\Phi}\left(x \chi_{A}\right)-I_{\Phi}\left(x \chi_{A \backslash A_{1}}\right) \\
& \geq(1+\eta) \Phi\left(\alpha_{1}\right) \mu(T)-\Phi\left(\alpha_{1}\right) \mu\left(A \backslash A_{1}\right) \\
& >\eta \Phi\left(\alpha_{\Phi}\right) \mu(T) .
\end{aligned}
$$

Applying Lemma 2 with $w=\alpha_{1}$ we get

$$
I_{\Phi}((x+y) / 2) \leq 1-\frac{\gamma}{2} I_{\Phi}\left(x \chi_{A_{1}}\right) \leq 1-\gamma \eta \Phi\left(\alpha_{\Phi}\right) \mu(T) / 2 .
$$

Consequently, Lemma $4(\mathrm{~b})$ yields $\|(x+y) / 2\|_{\Phi} \leq 1-q$ for some $q=$ $q\left(\gamma \eta \Phi\left(\alpha_{\Phi}\right) \mu(T) / 2\right)>0$. Therefore $\delta_{L_{\Phi}(\mu)}^{\perp}(a) \geq q>0$, so $\varepsilon_{0}^{\perp}\left(L_{\Phi}(\mu)\right) \leq u_{1}$.

(II.3) First note that $u_{2}<1$. Indeed, setting $z=a_{\Phi} \chi_{T}$, we get $I_{\Phi}(z)=0$ and $I_{\Phi}(z / \lambda) \leq 1$ for some $\lambda<1$, because $\Phi \in \Delta_{2}^{l}$ and consequently $b_{\Phi}=\infty$. Hence $\|z\|_{\Phi} \leq \lambda<1$.

We show that $\varepsilon_{0}^{\perp}\left(L_{\Phi}(\mu)\right) \geq u_{2}$. Let $\varepsilon>0$ and take $T_{0} \subset T, T_{0} \in \Sigma$ such that $\mu(T)-\varepsilon<\mu\left(T_{0}\right)<\mu(T)$. By assumption $\Phi \in \Delta_{2}^{l}$, hence $b_{\Phi}=\infty$. Then 
there exists $b>0$ such that $\Phi(b) \mu\left(T \backslash T_{0}\right)=1$. Define

$$
x=a_{\Phi} \chi_{T_{0}}+b \chi_{T \backslash T_{0}}, \quad y=b \chi_{T \backslash T_{0}} .
$$

Then $\|x\|_{\Phi}=\|y\|_{\Phi}=\|(x+y) / 2\|_{\Phi}=1$. Hence $\delta_{L_{\Phi}(\mu)}^{\perp}\left(a_{0}\right)=0$, where $a_{0}=\left\|a_{\Phi} \chi_{T_{0}}\right\|_{\Phi}$. Since $\varepsilon>0$ is arbitrary, we conclude that $\varepsilon_{0}^{\perp}\left(L_{\Phi}(\mu)\right) \geq u_{2}$.

It follows from Lemmas $4(\mathrm{a})$ and $5(\mathrm{a})$ that, under the corresponding assumptions, for every $a>0$ there is $\sigma(a)>0$ such that for any $x \in L_{\Phi}(\mu)$ (or $x \in l_{\Phi}$ ),

$$
\|x\|_{\Phi} \geq a \Rightarrow I_{\Phi}(x) \geq \sigma(a) .
$$

Hence, defining

$$
\sigma(a)=\inf \left\{I_{\Phi}(x):\|x\|_{\Phi} \geq a\right\}
$$

we get $\sigma(a)>0$ for each $a>0$. Moreover, the implications $\|u\|_{\Phi} \leq 1 \Rightarrow$ $I_{\Phi}(u) \leq\|u\|_{\Phi}$ and $\|u\|_{\Phi}>1 \Rightarrow I_{\Phi}(u)>\|u\|_{\Phi}$ yield

$$
\sigma(a) \leq a \text { for any } a \in(0,1], \quad \sigma(a) \geq a \text { for any } a>1 .
$$

REMARK 4. The upper estimate of $\varepsilon_{0}^{\perp}\left(L_{\Phi}(\mu)\right)$ in Theorem 2(II.2c) is, in some sense, optimal. Note that Theorem 2(II.2c) can be proved similarly for $u_{1}^{\sigma}=\sup \left\{u \geq 0: \sigma(u) \leq \Phi\left(\alpha_{\Phi}\right) \mu(T)\right\}$ in place of $u_{1}$, where $\sigma(\cdot)$ is from (6). On the other hand, the implication (5) is satisfied with $\sigma_{0}(u)=1 / f_{\Phi}(1 / u)$ (Lemma 6). Furthermore, by the definition of $f_{\Phi}, \sigma_{0}(\cdot)$ is the greatest possible function satisfying (5). Hence $u_{1}^{\sigma_{0}}=\sup \left\{u \geq 0: \sigma_{0}(u) \leq \Phi\left(\alpha_{\Phi}\right) \mu(T)\right\}$ $=1 / f_{\Phi}^{-1}\left(1 / \Phi\left(\alpha_{\Phi}\right) \mu(T)\right)$ is an optimal upper estimate for $\varepsilon_{0}^{\perp}\left(\bar{L}_{\Phi}(\mu)\right)$.

REMARK 5. It follows from Theorem 2 (case (II.2b)) that $\varepsilon_{0}^{\perp}\left(L_{\Phi}(\mu)\right) \in$ $\left[\Phi\left(\alpha_{\Phi}\right) \mu(T) / 2,1\right]$ whenever $\Phi\left(\alpha_{\Phi}\right) \mu(T) \in[1,2)$. Furthermore, in the class of Orlicz spaces $L_{\Phi}(\mu)$ generated by $\Phi \in \Delta_{2}^{l}$ with $\Phi\left(\alpha_{\Phi}\right) \mu(T) \in[1,2)$ the upper estimate of $\varepsilon_{0}^{\perp}\left(L_{\Phi}(\mu)\right)$ cannot be improved. Indeed, let us show that for each $\varepsilon>0$ there exists an Orlicz function $\Phi_{\varepsilon}$ with $\Phi_{\varepsilon}\left(\alpha_{\Phi_{\varepsilon}}\right) \mu(T) \in[1,2)$ and $\varepsilon_{0}^{\perp}\left(L_{\Phi_{\varepsilon}}(\mu)\right) \geq 1-\varepsilon$. Let $\varepsilon>0$ and let an Orlicz function $\Phi$ satisfy $\Phi\left(\alpha_{\Phi}\right) \mu(T) \in[1,2)$. Take $T_{1}^{\varepsilon} \in \Sigma$ with $\Phi\left(\alpha_{\Phi}\right) \mu\left(T_{1}^{\varepsilon}\right)=1-\varepsilon$. Since $\Phi \in \Delta_{2}^{l}$, we have $b_{\Phi}=\infty$. Hence there exists a set $T_{2}^{\varepsilon} \in \Sigma$ with $T_{2}^{\varepsilon} \subset T \backslash T_{1}^{\varepsilon}$ and a number $a_{\varepsilon}>\alpha_{\Phi}$ such that $\Phi\left(a_{\varepsilon}\right) \mu\left(T_{2}^{\varepsilon}\right)=\varepsilon$. Take $b_{\varepsilon}>0$ with $\Phi\left(b_{\varepsilon}\right) \mu\left(T_{2}^{\varepsilon}\right)=1$. Define

$$
\Phi_{\varepsilon}(u)= \begin{cases}\Phi(u) & \text { if } u \leq a_{\varepsilon} \text { or } u \geq b_{\varepsilon}, \\ \frac{\Phi\left(b_{\varepsilon}\right)-\Phi\left(a_{\varepsilon}\right)}{b_{\varepsilon}-a_{\varepsilon}} u+\frac{b_{\varepsilon} \Phi\left(a_{\varepsilon}\right)-a_{\varepsilon} \Phi\left(b_{\varepsilon}\right)}{b_{\varepsilon}-a_{\varepsilon}} & \text { if } u \in\left(a_{\varepsilon}, b_{\varepsilon}\right) .\end{cases}
$$

Clearly, $\Phi_{\varepsilon}\left(\alpha_{\Phi_{\varepsilon}}\right) \mu(T) \in[1,2)$. It is also easy to see that $\varepsilon_{0}^{\perp}\left(L_{\Phi_{\varepsilon}}(\mu)\right) \geq 1-\varepsilon$ by taking $x=\alpha_{\Phi_{\varepsilon}} \chi_{T_{1}^{\varepsilon}}+a_{\varepsilon} \chi_{T_{2}^{\varepsilon}}$ and $y=b_{\varepsilon} \chi_{T_{2}^{\varepsilon}}$.

TheOrem 3. Let $b_{\Phi}$ and $\sigma(\cdot)$ be as in (3) and (6), respectively. Then:

(i) $\varepsilon_{0}^{\perp}\left(l_{\Phi}\right)=1$ whenever $\Phi \notin \delta_{2}$ or $\Phi^{*} \notin \delta_{2}$ or $\Phi\left(b_{\Phi}\right) \leq 1 / 2$. 
(ii) $\varepsilon_{0}^{\perp}\left(l_{\Phi}\right) \in\left[1-\Phi\left(b_{\Phi}\right), u_{1}\right]$ if $\Phi\left(b_{\Phi}\right) \in(1 / 2,1), \Phi \in \delta_{2}, \Phi^{*} \in \delta_{2}$, where $u_{1}=\sup \left\{u \geq 0: \sigma(u) \leq 1-\Phi\left(b_{\Phi}\right)\right\}$.

(iii) $\varepsilon_{0}^{\perp}\left(l_{\Phi}\right)=0$ whenever $\Phi \in \delta_{2}, \Phi^{*} \in \delta_{2}$ and $\Phi\left(b_{\Phi}\right) \geq 1$.

Proof. (i) If $\Phi \notin \delta_{2}$ or $\Phi^{*} \notin \delta_{2}$, then $l_{\Phi}$ is not reflexive, and Theorem 1 yields $\varepsilon_{0}^{\perp}\left(l_{\Phi}\right)=1$. Suppose that $\Phi\left(b_{\Phi}\right) \leq 1 / 2$. Let $x=b_{\Phi} e_{1}+b_{\Phi} e_{2}$ and $y=$ $b_{\Phi} e_{1}$. Then $I_{\Phi}(x) \leq 1$ and $I_{\Phi}(x / \lambda)=\infty$ for every $0<\lambda<1$. Thus $\|x\|_{\Phi}=1$. Similarly $\|y\|_{\Phi}=1=\|(x+y) / 2\|_{\Phi}=\left\|x \chi_{A}\right\|_{\Phi}$, where $A=\operatorname{supp} x \div \operatorname{supp} y$, which finishes the proof.

(ii) Suppose that $\Phi \in \delta_{2}, \Phi^{*} \in \delta_{2}$ and $\Phi\left(b_{\Phi}\right) \in(1 / 2,1)$. Note that $1-\Phi\left(b_{\Phi}\right) \leq u_{1} \leq 1$. Indeed, suppose that $u_{1}<1-\Phi\left(b_{\Phi}\right)$, and take $u_{0}$ with $u_{1}<u_{0}<1-\Phi\left(b_{\Phi}\right)$. Then $\sigma\left(u_{0}\right)>1-\Phi\left(b_{\Phi}\right)$, and consequently, by $(7)$, we get a contradiction $1-\Phi\left(b_{\Phi}\right)<\sigma\left(u_{0}\right) \leq u_{0}<1-\Phi\left(b_{\Phi}\right)$. Assume that $u_{1}>1$. Then there are $\delta>0$ and $u_{\delta}>1+\delta$ with $\sigma\left(u_{\delta}\right) \leq 1-\Phi\left(b_{\Phi}\right)$. Hence, by (7), we get a contradiction $1+\delta<u_{\delta} \leq \sigma\left(u_{\delta}\right) \leq 1-\Phi\left(b_{\Phi}\right)$.

We now prove the lower bound. Since $\Phi\left(b_{\Phi}\right)>1 / 2$, we have $1-\Phi\left(b_{\Phi}\right)<$ $1 / 2$, and consequently there is $c>0$ such that $\Phi(c)=1-\Phi\left(b_{\Phi}\right)$. Let

$$
x=b_{\Phi} e_{1}+c e_{2}, \quad y=b_{\Phi} e_{1} .
$$

Then $\|x\|_{\Phi}=\|y\|_{\Phi}=1=\|(x+y) / 2\|_{\Phi}$. Moreover, setting $A=\operatorname{supp} x \div$ supp $y$, we get $I_{\Phi}\left(x \chi_{A} / \Phi(c)\right) \geq 1$, hence $\left\|x \chi_{A}\right\|_{\Phi} \geq \Phi(c)=1-\Phi\left(b_{\Phi}\right)$. Thus $\delta_{l_{\Phi}}^{\perp}\left(1-\Phi\left(b_{\Phi}\right)\right)=0$.

To prove the upper bound suppose that $a>u_{1}$. Then $\sigma(a)>1-$ $\Phi\left(b_{\Phi}\right)$. Let $x, y \in S\left(l_{\Phi}\right)$ be such that $\left\|x \chi_{A}\right\|_{\Phi} \vee\left\|y \chi_{A}\right\|_{\Phi} \geq a$, where $A=$ $\operatorname{supp} x \div \operatorname{supp} y$. Without loss of generality we may assume that $\left\|x \chi_{A_{1}}\right\|_{\Phi}=$ $\left\|x \chi_{A}\right\|_{\Phi} \geq a$, where $A_{1}=\operatorname{supp} x \backslash \operatorname{supp} y$. Take $\sigma_{1}>0$ such that $\sigma(a)>1-$ $\Phi\left(\left(1-\sigma_{1}\right) b_{\Phi}\right)$. The definition (6) implies $I_{\Phi}\left(x \chi_{A_{1}}\right) \geq \sigma(a)>1-\Phi\left(\left(1-\sigma_{1}\right) b_{\Phi}\right)$. Then Lemma 3 applied with $w=b_{\Phi}$ yields

$$
I_{\Phi}((x+y) / 2) \leq 1-\frac{\gamma}{2} I_{\Phi}\left(x \chi_{A_{1}}\right) \leq 1-\frac{\gamma}{2}\left(1-\Phi\left(b_{\Phi}\right)\right) .
$$

Moreover, $|x(i)| \leq\left(1-\sigma_{1}\right) b_{\Phi}$ for each $i \in \mathbb{N} \backslash A_{1}$, since otherwise $1 \geq$ $I_{\Phi}\left(x \chi_{A_{1}}\right)+I_{\Phi}\left(x \chi_{\mathbb{N} \backslash A_{1}}\right)>1$. Consequently, $\frac{x+y}{2}(i) \leq b_{\Phi} / 2$ for $i \in A_{1}$ and $\frac{x+y}{2}(i) \leq\left(2-\sigma_{1}\right) b_{\Phi} / 2$ for $i \in \mathbb{N} \backslash A_{1}$. Taking $\sigma_{2}=\min \left\{1 / 2, \sigma_{1} / 2\right\}$, and applying Lemma $5(\mathrm{~b})$ with $q=q\left(\sigma_{2}, \frac{\gamma}{2}\left(1-\Phi\left(b_{\Phi}\right)\right)\right)$ and inequality (8), we conclude that $\|(x+y) / 2\|_{\Phi} \leq 1-q$. Thus $\delta_{l_{\Phi}}^{\perp}(a) \geq q$. Since $a>u_{1}$ was arbitrary, we conclude that $\varepsilon_{0}^{\perp}\left(l_{\Phi}\right) \leq u_{1}$.

(iii) By the assumptions and $\left[20\right.$, Theorem 3], we get $l_{\Phi} \in\left(\mathrm{UC}^{\perp}\right)$, hence $\varepsilon_{0}^{\perp}\left(l_{\Phi}\right)=0$.

Note that we cannot find constructively the best possible function $\sigma(\cdot)$ in Theorem 3.2 as we do in Theorem 2(II.2.c) (see also Remark 4). If we take $f_{\Phi}^{0}(u)=\sup _{0<v \leq b_{\Phi}} \Phi(u v) / \Phi(v)$, then $f_{\Phi}^{0}(\cdot)$ is not finite-valued even in 
the case when $\Phi \in \delta_{2}$. Consequently, the result analogous to Lemma 6 is not valid in the sequence case when $b_{\Phi}<\infty$.

Applying Theorem 2(II.2.b) or Theorem 3(i) and criteria for superreflexivity of Orlicz spaces we conclude immediately that the converse of Theorem 1 is not true in general.

COROLlary 1. There exists an infinite-dimensional superreflexive Köthe space $E$ with $\varepsilon_{0}^{\perp}(E)=1$.

REMARK 6. Recall that any Banach space with $\varepsilon_{0}(X)<2$ is superreflexive (see Remark 2). Similarly to Corollary 1, there is a superreflexive Banach space $X$ with $\varepsilon_{0}(X)=2$. It is enough to take $X=L_{\Phi}(\mu)$ satisfying the assumptions of Theorem 2(II.2.b). To show that $\varepsilon_{0}\left(L_{\Phi}(\mu)\right)=2$ it is enough to consider elements $x$ and $y$ as in the relevant proof (case A). Combining this with Remark 2 in Section 2.1 we see that $\varepsilon_{0}^{\perp}(E)$ plays the same role with regard to superreflexivity in Köthe spaces as does $\varepsilon_{0}(X)$ for superreflexivity in Banach spaces.

2.3. Köthe-Bochner spaces. Let us define the type of spaces to be considered hereafter. For a real Banach space $\left(X,\|\cdot\|_{X}\right)$, denote by $M(T, X)$, or just by $M(X)$, the family of strongly measurable functions $f: T \rightarrow X$, where functions which are equal $\mu$-almost everywhere are identified. Given a Köthe space $E$ (see Definition 1) define

$$
\widetilde{x}(\cdot)=\|x(\cdot)\|_{X}, \quad E(X)=\{x \in M(X): \widetilde{x} \in E\} .
$$

Then $E(X)$ equipped with the norm

$$
\|x\|_{E(X)}=\|\widetilde{x}\|_{E}
$$

becomes a Banach space and it is called a Köthe-Bochner space.

We shall consider Köthe-Bochner space $E(X)$, where $E=E\left(T, \Sigma_{1}, \mu_{1}\right)$ and $X=X\left(S, \Sigma_{2}, \mu_{2}\right)$ are Köthe spaces over the measure spaces $\left(T, \Sigma_{1}, \mu_{1}\right)$ and $\left(S, \Sigma_{2}, \mu_{2}\right)$. Then we may view an element $x \in E(X)$ as a function $x: T \times S \rightarrow \mathbb{R}$ such that $x(t, \cdot) \in X$ for each $t \in T$ and the function $t \mapsto$ $\|x(t, \cdot)\|_{X}$ is an element of $E$. Clearly, $\operatorname{supp} x=\{(t, s) \in T \times S: x(t, s) \neq 0\}$.

In order to study orthogonal uniform convexity in the spaces $E(X)$ we notice that this property can be considered not only in Köthe spaces but more generally in normed function spaces which have the so-called semiKöthe property.

Definition 3. A normed function space $E \subset L^{0}$ is a semi-Köthe space $(E \in(\mathrm{sK}))$ if for any $x, y \in E$ we have $x \chi_{A_{x y}} \in E$, where $A_{x y}=\operatorname{supp} x \div$ supp $y$.

Remark 7. Clearly, if $E$ is a Köthe space, then $E \in(\mathrm{sK})$. Note also that the converse is not true. Let $\left(E,\|\cdot\|_{E}\right)$ be a Köthe space and $E_{1} \subset E$ 
be the set of all simple functions. Then $E_{1} \in(\mathrm{sK})$ and $E_{1}$ is not a Köthe space, since given $x \in E_{1}$ it is easy to find $y \in L^{0}$ such that $|y| \leq|x|$ and $y$ is not a simple function. Note that $E_{1}$ is not complete. However, there is also an example of a Banach (complete) function space $E$ with $E \in(\mathrm{sK})$ which is not a Köthe space. Indeed, if a Köthe space $E$ is not reflexive, then it contains a subspace $X$ which is isomorphic to $c_{0}$ or to $l^{1}$. By the construction of $X$ we conclude that there exists a sequence $\left(u_{n}\right)_{n=1}^{\infty} \subset E$ with pairwise disjoint supports such that for every $x \in X$ there exists a sequence $\left(t_{n}^{x}\right)_{n=1}^{\infty} \subset l^{\infty}$ such that $x=\sum_{n=1}^{\infty} t_{n}^{x} u_{n}$ (see [3, Theorem 4], [23, Theorem 1.c.5] and [27, Theorem 5.16]). Hence $X$ is a semi-Köthe space. Moreover, it is complete. On the other hand, $X$ does not satisfy condition (i) from the definition of the Köthe space.

The following question arises:

Question. Let $E$ be a semi-Köthe space over the measure space $(T, \Sigma, \mu)$. Does there exist a subalgebra $\Sigma_{0} \subset \Sigma$ such that each $x \in E$ is $\Sigma_{0^{-}}$ measurable and the space $E_{0}$ defined to be $E$ considered over $\left(T, \Sigma_{0}, \mu / \Sigma_{0}\right)$ is a Köthe space?

The answer is negative in general. It is enough to take the space $E_{1}$ from Remark 7. Indeed, the only subalgebra $\Sigma_{0} \subset \Sigma$ such that each $x \in E_{1}$ is $\Sigma_{0}$-measurable is the whole $\Sigma$.

Similarly, a negative answer can be deduced if we consider the space $X$ from Remark 7. Then the smallest subalgebra $\Sigma_{0} \subset \Sigma$ such that each $x \in X$ is $\Sigma_{0}$-measurable, is defined by $\Sigma_{0}=\left\{S \in \Sigma: S=\bigcup_{n \in A} T_{n}^{a}\right.$, $A \subset \mathbb{N}, a \in \mathbb{R}\}$, where $T_{n}=\operatorname{supp} u_{n}$ and $T_{n}^{a}=\left\{t \in T_{n}:\left|u_{n}(t)\right|<a\right\}$ for each $a \in \mathbb{R}$. Clearly, $X_{0}=X$ considered over $\left(T, \Sigma_{0}, \mu / \Sigma_{0}\right)$ is not a Köthe space, because $X_{0}$ does not satisfy condition (i) from the definition of the Köthe space.

Note that $E(X) \in(\mathrm{sK})$. Indeed, given $x, y \in E(X)$ and setting $F=$ $\operatorname{supp} x \backslash \operatorname{supp} y$ and $G(t)=\operatorname{supp} x(t) \backslash \operatorname{supp} y(t) \subset S$, we have $x \chi_{F}(t)=$ $x(t) \chi_{G(t)} \in X$ for each $t \in T$, since $x(t), y(t) \in X$ and $X \in(\mathrm{sK})$. Furthermore, $\left|x(t) \chi_{G(t)}\right| \leq|x(t)| \mu_{2}$-a.e. in $S$ for each $t \in T$, hence $\left\|x(t) \chi_{G(t)}\right\|_{X} \leq$ $\|x(t)\|_{X}$ for each $t \in T$. Then the function $t \mapsto\left\|x(t) \chi_{G(t)}\right\|_{X}$ is an element of $E$. Consequently, $\left\|x \chi_{F}\right\|_{E(X)}=\|\| x(\cdot) \chi_{G(\cdot)}\left\|_{X}\right\|_{E}$ and the orthogonal uniform convexity is well defined in the space $E(X)$. However, the natural question arises.

Question. Given Köthe spaces $E=E\left(T, \Sigma_{1}, \mu_{1}\right)$ and $X=X\left(S, \Sigma_{2}, \mu_{2}\right)$, can the space $E(X)$ be considered as another Köthe space?

The answer was given by Bukhvalov in [5] in a more general case.

Denote by $(P, \Sigma, \mu)$ the product measure space $\left(T \times S, \Sigma_{1} \otimes \Sigma_{2}, \mu_{1} \times \mu_{2}\right)$. Let $E[X]$ be the space all $\mu$-measurable functions $K(t, s)(t \in T, s \in S)$ such 
that for each $K \in E[X]$ we have

(i) $K(t, \cdot) \in X$ for $\mu_{1}$-a.e. $t \in T$.

Then we define $\omega_{K}(t)=\|K(t, \cdot)\|_{X}$ for $\mu_{1}$-a.e. $t \in T$. If we suppose additionally that $\left(X,\|\cdot\|_{X}\right)$ is monotone complete, $X \in(\mathrm{MC})$, that is, $0 \leq x_{n} \uparrow$ $x \in X$ implies $\lim _{n \rightarrow \infty}\left\|x_{n}\right\|_{X}=\|x\|_{X}$, then $\omega_{K}(\cdot)$ is $\Sigma_{1}$-measurable $([5$, Theorem 1.1]). It is known that to get $\Sigma_{1}$-measurability of $\omega_{K}(\cdot)$ we cannot drop the assumption of monotone completeness of $X$. Then, if $X \in(\mathrm{MC})$, we may additionally assume in the definition of the space $E[X]$ that

(ii) the function $\omega_{K}(t)=\|K(t, \cdot)\|_{X}$ is an element of $E$.

Consequently, if we endow $E[X]$ with the norm $\|K\|_{E[X]}=\left\|\omega_{K}(\cdot)\right\|_{E}$, then $\left(E[X],\|\cdot\|_{E[X]}\right)$ satisfies conditions (i) and (ii) in the definition of a Köthe space. Since $E$ and $X$ are Banach spaces, so is $E[X]$ ([5, Theorem 1.5]). Moreover, we have

TheOrem $4\left(\left[5\right.\right.$, Theorem 2.2]). The spaces $\left(E[X],\|\cdot\|_{E[X]}\right)$ and $(E(X)$, $\left.\|\cdot\|_{E(X)}\right)$ are isomorphically isometric if and only if either $X$ is order continuous or the measure $\mu_{1}$ is purely atomic.

We want to thank Professor M. Mastyło for pointing out Bukhvalov's result.

For any $x \in E \backslash\{0\}$ set $\widehat{x}=x /\|x\|_{E}$. We shall need two lemmas.

LEMma 7. Let $x, y \in E \backslash\{0\}$. If $\left\|\widehat{x} \chi_{A}\right\|_{E} \vee\left\|\widehat{y} \chi_{A}\right\|_{E} \geq \varepsilon$, where $A=$ $\operatorname{supp} x \div \operatorname{supp} y$, and $\|x\|_{E} \wedge\|y\|_{E} \geq \eta\left(\|x\|_{E} \vee\|y\|_{E}\right)$, then

$$
\|x+y\|_{E} \leq\left(1-\eta \delta_{E}^{\perp}(\varepsilon)\right)\left(\|x\|_{E}+\|y\|_{E}\right) .
$$

The proof can be done the same way as in [13, Lemma 1.4].

LEMMA 8 ([11, Theorem 7]). $E \in(\mathrm{UM})$ if and only if for any $\varepsilon \in(0,1)$ there is $\eta(\varepsilon)>0$ such that $\left\|x \chi_{T \backslash A}\right\|_{E} \leq 1-\eta(\varepsilon)$ for any $x \in E$ with $x \geq 0$, $\|x\|_{E}=1$ and for any $A \in \Sigma$ such that $\left\|x \chi_{A}\right\|_{E} \geq \varepsilon$.

Theorem 5. Let $E$ and $X$ be Köthe spaces. Assume that $E$ is uniformly monotone. Then:

(i) $\varepsilon_{0}^{\perp}(X) \vee \varepsilon_{0}^{\perp}(E) \leq \varepsilon_{0}^{\perp}(E(X)) \leq \varepsilon_{0}^{\perp}(X)+\varepsilon_{0}^{\perp}(E)-\varepsilon_{0}^{\perp}(X) \varepsilon_{0}^{\perp}(E)$.

(ii) Both inequalities in (i) are equalities if and only if either $\varepsilon_{0}^{\perp}(X)$ or $\varepsilon_{0}^{\perp}(E)$ is in $\{0,1\}$. In particular:

(a) $E(X)$ is orthogonally uniformly convex if and only if both $E$ and $X$ are orthogonally uniformly convex.

(b) $\varepsilon_{0}^{\perp}(E(X))=\varepsilon_{0}^{\perp}(E)$ whenever $X$ is orthogonally uniformly convex.

(c) $\varepsilon_{0}^{\perp}(E(X))=\varepsilon_{0}^{\perp}(X)$ if $E$ is orthogonally uniformly convex. 
(iii) For any $\alpha, \eta \in(0,1)$ and $\varepsilon \in(\alpha \vee \eta, \alpha+\eta-\alpha \eta)$ there exists a twodimensional Köthe space $E$ such that $\varepsilon_{0}^{\perp}(E)=\eta$ and $\varepsilon_{0}^{\perp}(E(X))=\varepsilon$ whenever $\varepsilon_{0}^{\perp}(X)=\alpha$.

We shall apply some techniques and methods from the proof of $[13$, Theorem 1]. For any $x \in E(X)$ we write $\|x\|$ instead of $\|x\|_{E(X)}$ for simplicity.

Proof. (i) The lower bound is obvious. We prove the upper bound. Let $E=E\left(T, \Sigma_{1}, \mu_{1}\right)$ and $X=X\left(S, \Sigma_{2}, \mu_{2}\right)$. Set $\alpha=\varepsilon_{0}^{\perp}(X), \eta=\varepsilon_{0}^{\perp}(E)$ and $\varepsilon=\varepsilon_{0}^{\perp}(E(X))$. Take sequences $\left\{x_{n}\right\},\left\{y_{n}\right\}$ in $S(E(X))$ with $\left\|x_{n}+y_{n}\right\| \rightarrow 2$ and $\left\|x_{n} \chi_{F_{n}}\right\| \vee\left\|y_{n} \chi_{F_{n}}\right\| \rightarrow \varepsilon$, where $F_{n}=\operatorname{supp} x_{n} \div \operatorname{supp} y_{n}$. Then $\left\|x_{n} \chi_{F_{n}}\right\|=$ \|\|$x_{n}(\cdot) \chi_{G_{n}(\cdot)}\left\|_{X}\right\|_{E}$ and $G_{n}(t)=\operatorname{supp} x_{n}(t) \backslash \operatorname{supp} y_{n}(t) \subset S$ (see the introduction in Section 2.3). Let

$$
s_{n}(\cdot)=\left\|\left(x_{n}+y_{n}\right)(\cdot)\right\|_{X}, \quad S_{n}(\cdot)=\left\|x_{n}(\cdot)\right\|_{X}+\left\|y_{n}(\cdot)\right\|_{X} .
$$

We have $2 \leftarrow\left\|s_{n}\right\|_{E} \leq\left\|S_{n}\right\|_{E} \leq 2$. Take $\eta_{n} \downarrow 0$ and $\varepsilon_{n} \downarrow \alpha$ such that

$$
\frac{\left\|S_{n}\right\|_{E}-\left\|s_{n}\right\|_{E}}{\gamma_{n}} \rightarrow 0
$$

where $\gamma_{n}=\eta_{n} \delta_{X}^{\perp}\left(\varepsilon_{n}\right)$. Define

$$
\begin{aligned}
A_{n}\left(\eta_{n}\right) & =\left\{t \in T:\left\|x_{n}(t)\right\|_{X} \wedge\left\|y_{n}(t)\right\|_{X} \geq \eta_{n}\left(\left\|x_{n}(t)\right\|_{X} \vee\left\|y_{n}(t)\right\|_{X}\right)\right\}, \\
A_{n}^{\geq} & =\left\{t \in A_{n}\left(\eta_{n}\right):\left\|\widehat{x_{n}(t)} \chi_{G_{n}(t)}\right\|_{X} \vee\left\|\widehat{y_{n}(t)} \chi_{G_{n}(t)}\right\|_{X} \geq \varepsilon_{n}\right\}, \\
A_{n}^{<} & =A_{n}\left(\eta_{n}\right) \backslash A_{n}^{\geq},
\end{aligned}
$$

where $\widehat{x_{n}(t)}=x_{n}(t) /\left\|x_{n}(t)\right\|_{X}$. Applying Lemma 7 we get $s_{n}(\cdot) \chi_{A_{\bar{n}}^{>}} \leq$ $\left(1-\gamma_{n}\right) S_{n}(\cdot) \chi_{A_{n}} \cdot$ Clearly, $s_{n}(\cdot) \leq S_{n}(\cdot)$. Then

$$
\begin{aligned}
\left\|s_{n}\right\|_{E} & \leq\left\|S_{n}-\gamma_{n} S_{n} \chi_{A_{n}}\right\|_{E}=\left\|S_{n}-\gamma_{n}\left(S_{n}-S_{n} \chi_{T \backslash A_{n}}\right)\right\|_{E} \\
& \leq\left(1-\gamma_{n}\right)\left\|S_{n}\right\|_{E}+\gamma_{n}\left\|S_{n} \chi_{T \backslash A_{n}^{\geq}}\right\|_{E} .
\end{aligned}
$$

Consequently, by (9),

$$
2 \geq\left\|S_{n} \chi_{T \backslash A_{n}^{\geq}}\right\|_{E} \geq\left\|S_{n}\right\|_{E}-\frac{1}{\gamma_{n}}\left\{\left\|S_{n}\right\|_{E}-\left\|s_{n}\right\|_{E}\right\} \rightarrow 2 .
$$

In particular \|\|$x_{n}(\cdot)\left\|_{X} \chi_{T \backslash A_{n}}\right\|_{E} \rightarrow 1$. Consequently,

$$
\|\| x_{n}(\cdot)\left\|_{X} \chi_{A_{\bar{n}}}\right\|_{E} \rightarrow 0
$$

because otherwise applying uniform monotonicity of $E$ and Lemma 8 we would get a contradiction. Since $T \backslash A_{n}^{<}=\left[T \backslash A_{n}\left(\eta_{n}\right)\right] \cup A_{\bar{n}}^{\geq}$, we get

$$
\begin{aligned}
\left\|x_{n} \chi_{F_{n}}\right\| & =\|\| x_{n}(\cdot) \chi_{G_{n}(\cdot)}\left\|_{X}\right\|_{E} \\
& =\|\| x_{n}(\cdot) \chi_{G_{n}(\cdot)}\left\|_{X} \chi_{A_{n}^{<}}+\left(\varepsilon_{n}+1-\varepsilon_{n}\right)\right\| x_{n}(\cdot) \chi_{G_{n}(\cdot)}\left\|_{X} \chi_{T \backslash A_{n}}\right\|_{E}
\end{aligned}
$$




$$
\begin{aligned}
\leq & \| \varepsilon_{n}\left(\left\|x_{n}(\cdot)\right\|_{X} \chi_{A_{n}^{<}}+\left\|x_{n}(\cdot) \chi_{G_{n}(\cdot)}\right\|_{X} \chi_{T \backslash A_{n}^{<}}\right) \\
& +\left(1-\varepsilon_{n}\right)\left\|x_{n}(\cdot) \chi_{G_{n}(\cdot)}\right\|_{X} \chi_{T \backslash A_{n}^{<}} \|_{E} \\
\leq & \varepsilon_{n}\left\|x_{n}\right\|+\left(1-\varepsilon_{n}\right)\left(\|\| x_{n}(\cdot) \chi_{G_{n}(\cdot)}\left\|_{X} \chi_{T \backslash A_{n}\left(\eta_{n}\right)}\right\|_{E}\right. \\
& \left.+\|\| x_{n}(\cdot) \chi_{G_{n}(\cdot)}\left\|_{X} \chi_{A_{n}^{>}}\right\|_{E}\right) \\
\leq & \varepsilon_{n}+\left(1-\varepsilon_{n}\right)\|\| x_{n}(\cdot) \chi_{G_{n}(\cdot)}\left\|_{X} \chi_{T \backslash A_{n}\left(\eta_{n}\right)}\right\|_{E}+\|\| x_{n}(\cdot)\left\|_{X} \chi_{A_{n}^{\frac{\geq}{n}}}\right\|_{E} .
\end{aligned}
$$

Without loss of generality we may assume that $\left\|x_{n} \chi_{F_{n}}\right\|=\left\|x_{n} \chi_{F_{n}}\right\| \vee$ $\left\|y_{n} \chi_{F_{n}}\right\|$ for any $n \in \mathbb{N}$. Hence, by (10) we get

$$
\begin{aligned}
\|\| x_{n}(\cdot) \chi_{G_{n}(\cdot)}\left\|_{X} \chi_{T \backslash A_{n}\left(\eta_{n}\right)}\right\|_{E} & \geq \frac{\left\|x_{n} \chi_{F_{n}}\right\|-\varepsilon_{n}-\|\| x_{n}(\cdot)\left\|_{X} \chi_{A_{n}}\right\|_{E}}{1-\varepsilon_{n}} \\
& \rightarrow \frac{\varepsilon-\alpha}{1-\alpha} .
\end{aligned}
$$

Let $T \backslash A_{n}\left(\eta_{n}\right)=B_{n} \cup C_{n}$, where

$$
\begin{aligned}
& B_{n}=\left\{t \in T \backslash A_{n}\left(\eta_{n}\right):\left\|x_{n}(t)\right\|_{X}=\left\|x_{n}(t)\right\|_{X} \wedge\left\|y_{n}(t)\right\|_{X}\right\}, \\
& C_{n}=\left(T \backslash A_{n}\left(\eta_{n}\right)\right) \backslash B_{n} .
\end{aligned}
$$

Set

$$
u_{n}=\left\|x_{n}(\cdot)\right\|_{X}, \quad v_{n}=\left\|y_{n}(\cdot)\right\|_{X} \chi_{T \backslash C_{n}} .
$$

We have \|\|$y_{n}(\cdot)\left\|_{X} \chi_{C_{n}}\right\|_{E} \rightarrow 0$, and consequently, as $\left\|S_{n}\right\|_{E} \rightarrow 2$,

$$
\left\|u_{n}+v_{n}\right\|_{E} \rightarrow 2 \text {. }
$$

Since \|\|$x_{n}(\cdot) \chi_{G_{n}(\cdot)}\left\|_{X} \chi_{B_{n}}\right\|_{E} \rightarrow 0$, from (11) it follows that

$$
\liminf \|\| x_{n}(\cdot) \chi_{G_{n}(\cdot)}\left\|\chi_{X C_{n}}\right\|_{E} \geq \frac{\varepsilon-\alpha}{1-\alpha} .
$$

Hence, by (12), setting $D_{n}=\operatorname{supp} u_{n} \div \operatorname{supp} v_{n}$, we get

$$
\eta \geq \liminf \left\|u_{n} \chi_{D_{n}}\right\|_{E}=\liminf \|\| x_{n}(\cdot)\left\|_{X} \chi_{C_{n}}\right\|_{E} \geq \frac{\varepsilon-\alpha}{1-\alpha} .
$$

(ii) follows immediately from (i).

(iii) Let $\alpha, \eta \in(0,1)$ and $\varepsilon \in(\alpha \vee \eta, \alpha+\eta-\alpha \eta)$. Let $E=\mathbb{R}^{2}$. We can (and do) define the norm $\|\cdot\|_{E}$ in $E$ such that the positive part of the unit sphere $S(E)^{+}$will be the set (see Figure 1):

$$
S(E)^{+}=(0,1)-(1,1) \frown\left(\frac{1}{\varepsilon}, \frac{\alpha}{\varepsilon}\right) \frown\left(\frac{1}{\eta}, 0\right),
$$

where the symbols $\lambda-\mu$ and $\lambda \frown \mu$ denote a straight line and a strictly rotund part of $S(E)$ for any $\lambda, \mu$ in the unit sphere of $E$. Indeed, given a convex, absorbing and balanced set $A$, the Minkowski functional $K_{A}$ of $A$ defined by $K_{A}(x)=\inf \{\alpha>0: x / \alpha \in A\}, x \in E$, defines a norm in $E$ by 


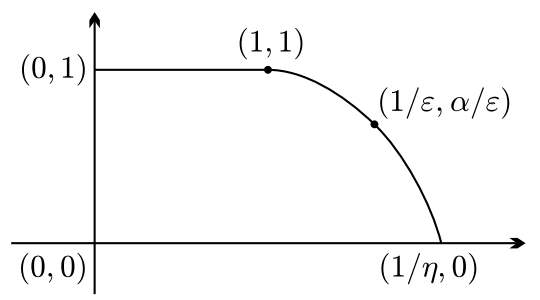

Fig. 1

the formula $\|x\|_{E}=K_{A}(x)$. Moreover, since $\operatorname{dim} E<\infty$, the boundary of $A$ is equal to the unit sphere $\left(S(E),\|\cdot\|_{E}\right)$.

Clearly, $\varepsilon_{0}^{\perp}(E)=\|(1,0)\|_{E}=\frac{1}{1 / \eta}=\eta$. Suppose that $\varepsilon_{0}^{\perp}(X)=\alpha$. Then we find $u_{n}, v_{n} \in X$ with $\left\|u_{n}\right\|_{X}=\left\|v_{n}\right\|_{X}=1,\left\|u_{n}+v_{n}\right\|_{X} \rightarrow 2$ and $\left\|u_{n} \chi_{A_{n}}\right\|_{X} \vee\left\|v_{n} \chi_{A_{n}}\right\|_{X} \rightarrow \alpha$, where $A_{n}=\operatorname{supp} u_{n} \div \operatorname{supp} v_{n}$. We may assume that $\left\|u_{n} \chi_{A_{n}}\right\|_{X}=\left\|u_{n} \chi_{A_{n}}\right\|_{X} \vee\left\|v_{n} \chi_{A_{n}}\right\|_{X}$ for any $n \in \mathbb{N}$. Let $x_{n}=\left(v_{n}, u_{n}\right)$ and $y_{n}=\left(0, v_{n}\right)$. Then $\left\|x_{n}\right\|=\left\|y_{n}\right\|=1$. Moreover, setting $F_{n}=\operatorname{supp} x_{n} \div \operatorname{supp} y_{n}$, we get $\left\|x_{n} \chi_{F_{n}}\right\| \rightarrow\|(1, \alpha)\|_{E}=\varepsilon$. On the other hand, $\left\|x_{n}+y_{n}\right\|=\left\|\left(\left\|v_{n}\right\|_{X},\left\|u_{n}+v_{n}\right\|_{X}\right)\right\|_{E} \rightarrow\|(1,2)\|_{E}=2$. Hence $\varepsilon_{0}^{\perp}(E(X)) \geq \varepsilon$. Suppose now that there are $x_{n}, y_{n} \in E(X)$ with $\left\|x_{n}\right\|=\left\|y_{n}\right\|=1 \leftarrow\left\|\left(x_{n}+y_{n}\right) / 2\right\|$ and $\left\|x_{n} \chi_{F_{n}}\right\| \vee\left\|y_{n} \chi_{F_{n}}\right\| \rightarrow \varepsilon^{\prime}$. Then, without loss of generality, $x_{n}=\left(u_{n}, v_{n}\right), y_{n}=\left(w_{n}, z_{n}\right)$ with

$$
\begin{gathered}
\left\|u_{n}\right\|_{X} \rightarrow u, \quad\left\|v_{n}\right\|_{X} \rightarrow v, \quad\left\|w_{n}\right\|_{X} \rightarrow w, \quad\left\|z_{n}\right\|_{X} \rightarrow z, \\
\left\|\left(u_{n}+w_{n}\right) / 2\right\|_{X} \rightarrow r, \quad\left\|u_{n} \chi_{A_{n}}\right\|_{X} \vee\left\|w_{n} \chi_{A_{n}}\right\|_{X} \rightarrow p, \\
\left\|\left(v_{n}+z_{n}\right) / 2\right\|_{X} \rightarrow s, \quad\left\|v_{n} \chi_{B_{n}}\right\|_{X} \vee\left\|z_{n} \chi_{B_{n}}\right\|_{X} \rightarrow q,
\end{gathered}
$$

where $A_{n}=\operatorname{supp} u_{n} \div \operatorname{supp} w_{n}$ and $B_{n}=\operatorname{supp} v_{n} \div \operatorname{supp} z_{n}$, so that

$$
\|(u, v)\|_{E}=1=\|(w, z)\|_{E},\|(p, q)\|_{E}=\varepsilon^{\prime}
$$

and

$$
1=\|(r, s)\|_{E} \leq\left\|\frac{1}{2}(u, v)+\frac{1}{2}(w, z)\right\|_{E} \leq 1 .
$$

Consequently, $(u, v),(w, z) \in(0,1)-(1,1)$, hence $v=z=1=s$ and $u, w \leq 1$. Then $q \leq \alpha$ and $p \leq 1$, which gives

$$
\varepsilon^{\prime}=\|(p, q)\|_{E} \leq\|(1, \alpha)\|_{E}=\varepsilon
$$

Acknowledgments. 1. This paper was finished in November 2002 while the first-named author visited the Institute of Mathematics of the Polish Academy of Science as a fellow of a scholarship of the Foundation of Polish Science. He wants to express his gratitude to Professor S. Rolewicz for kind hospitality and numerous valuable consultations, and to the Foundation of Polish Science for generous financial support. He also wants to thank 
Professor A. Pełczyński and Professor P. Mankiewicz for giving inspiration to new studies.

2. We wish to thank the referee for his valuable remarks which led to substantial improvements of the paper.

\section{References}

[1] A. G. Aksoy and M. A. Khamsi, Nonstandard Methods in Fixed Point Theory, Springer, 1990.

[2] G. Alherk and H. Hudzik, Uniformly non- $l_{n}^{(1)}$ Musielak-Orlicz spaces of Bochner type, Forum Math. 1 (1989), 403-410.

[3] C. Bessaga and A. Pełczyński, On bases and uconditional convergence of series in Banach spaces, Studia Math. 17 (1958), 151-164.

[4] G. Birkhoff, Lattice Theory, Amer. Math. Soc., Providence, RI, 1967.

[5] A. V. Bukhvalov, Spaces with mixed norm, Vestnik Leningrad. Univ. Mat. Mekh. Astronom. 19 (1973), no. 4, 5-12 (in Russian).

[6] S. S. Chen, Geometry of Orlicz spaces, Dissertationes Math. 356 (1996).

[7] J. A. Clarkson, Uniformly convex spaces, Trans. Amer. Math. Soc. 40 (1936), 396414.

[8] T. Dominguez, H. Hudzik, G. López, M. Mastyło and B. Sims, Complete characterization of Kadec-Klee properties in Orlicz spaces, Houston J. Math. 29 (2004), 1027-1044.

[9] K. Goebel and W. A. Kirk, Topics in Metric Fixed Point Theory, Cambridge Univ. Press, Cambridge, 1990.

[10] H. Hudzik, Uniform convexity of Musielak-Orlicz spaces with Luxemburg's norm, Comment. Math. 23 (1983), 23-30.

[11] H. Hudzik, A. Kamińska and M. Mastyło, Monotonicity and rotundity properties in Banach lattices, Rocky Mountain J. Math. 30 (2000), 933-950.

[12] H. Hudzik, A. Kamińska and J. Musielak, On the convexity coefficient of Orlicz spaces, Math. Z. 197 (1988), 291-295.

[13] H. Hudzik and T. Landes, Characteristic of convexity of Köthe function spaces, Math. Ann. 294 (1992), 117-124.

[14] -, -, Packing constant in Orlicz spaces equipped with the Luxemburg norm, Boll. Un. Mat. Ital. (7) 9-A (1995), 225-237.

[15] R. C. James, Uniformly non-square Banach spaces, Ann. of Math. 80 (1964), 542550 .

[16] A. Kamińska, Uniform rotundity of Musielak-Orlicz sequence spaces, J. Approx. Theory 47 (1986), 302-322.

[17] L. V. Kantorovich and G. P. Akilov, Functional Analysis, Nauka, Moscow, 1984 (in Russian).

[18] P. Kolwicz, The property ( $\beta$ ) of Orlicz Bochner sequence spaces, Comment. Math. Univ. Carolin. 42 (2001), 119-132.

[19] - On property $(\beta)$ in Banach lattices, Calderón-Lozanovskiu and Orlicz-Lorentz spaces, Proc. Indian Acad. Sci. (Math. Sci.) 111 (2001), 319-336.

[20] - Orthogonal uniform convexity in Köthe spaces and Orlicz spaces, Bull. Polish Acad. Sci. Math. 50 (2002), 395-412.

[21] - Property $(\beta)$ and orthogonal convexities in some class of Köthe sequence spaces, Publ. Math. Debrecen 63 (2004), 587-609. 
[22] C. A. Kottman, Packing and reflexivity in Banach spaces, Trans. Amer. Math. Soc. 150 (1970), 565-576.

[23] J. Lindenstrauss and L. Tzafriri, Classical Banach Spaces II, Springer, Berlin 1979.

[24] J. Musielak, Orlicz Spaces and Modular Spaces, Lecture Notes in Math. 1034, Springer, Berlin, 1983.

[25] D. Pallaschke and S. Rolewicz, Foundations of Mathematical Optimization, Math. Appl. 388, Kluwer, Dordrecht, 1997.

[26] S. Rolewicz, On $\Delta$-uniform convexity and drop property, Studia Math. 87 (1987), 181-191.

[27] H. H. Schaefer, Banach Lattices and Positive Operators, Springer, Berlin, 1974.

Institute of Mathematics

University of Technology

Piotrowo $3 \mathrm{a}$

60-965 Poznań, Poland

E-mail: kolwicz@math.put.poznan.pl
Institute of Mathematics Polish Academy of Sciences Śniadeckich 8 P.O. Box 21

00-956 Warszawa, Poland E-mail: rolewicz@impan.gov.pl

Received May 5, 2003

Revised version December 29, 2003 